\title{
Culturomics: bacterial species isolated in 3 healthy donors for faecal microbiota transplantation in Clostridium difficile infection
}

\author{
Luca Masucci, ${ }^{1}$ Gianluca Quaranta, ${ }^{1}$ Domenico Nagel, ${ }^{1}$ Sandra Primus, ${ }^{2}$ Lucio Romano, ${ }^{1}$ \\ Rosalia Graffeo, ${ }^{1}$ Gianluca Ianiro, ${ }^{3}$ Antonio Gasbarrini, ${ }^{3}$ Giovanni Cammarota, ${ }^{3}$ Maurizio Sanguinetti ${ }^{1}$ \\ ${ }^{1}$ Institute of Microbiology, Catholic University of the Sacred Heart, A. Gemelli Foundation, School of Medicine, Rome; \\ ${ }^{2}$ College for Healthcare Professions, Catholic University of the Sacred Heart, Claudiana, Bolzano; ${ }^{3}$ Internal Medicine, \\ Gastroenterology and Liver Unit, Catholic University of the Sacred Heart, School of Medicine, A. Gemelli Foundation, \\ Rome, Italy
}

\begin{abstract}
Summary
Background. Clostridium difficile infections are the main cause of nosocomial acquired diarrhea, because of prolonged antibiotic regimens. In the last years, mortality has increased due to recurrent infections caused by metronidazole and vancomicin resistant hypervirulent $C$. difficile strain 027. Faecal Microbiota Transplantation (FMT) is an infusion of faecal material obtained from healthy donors. This procedure reduces mortality in recurrent $C$. difficile infections (CDI). In this study we identified bacterial species obtained from donors' stool samples using culturomics.

Materials and methods. Three donors were selected for FMT in three recipients affected by CDI. Faces obtained for
\end{abstract}

\footnotetext{
Correspondence: Luca Masucci, Institute of Microbiology, Catholic University of the Sacred Heart, A. Gemelli Foundation, School of Medicine, L.go A. Gemelli 8, 00168 Rome, Italy.

Tel.: +39.06.30154218 - Fax: +39.06.3051152

E-mail: luca.masucci@policlinicogemelli.it

Key words: Culturomics; Faecal microbiota transplantation; Clostridium difficile.

Contribution: LM, GC, study concept and design; LM, GQ, DN, SP, LR, RG, laboratory work ; GC, GI, AG, clinical activity; LM, LR, MS, drafting of the manuscript.

Conflict of interest: the authors declare no potential conflict of interest.

Received for publication: 22 December 2016.

Revision received: 1 March 2017.

Accepted for publication: 1 March 2017.

(C) Copyright L. Masucci et al., 2017

Licensee PAGEPress, Italy

Microbiologia Medica 2017; 32:6510

doi:10.4081/mm.2017.6510

This article is distributed under the terms of the Creative Commons Attribution Noncommercial License (by-nc 4.0) which permits any noncommercial use, distribution, and reproduction in any medium, provided the original author(s) and source are credited.
}

FMT underwent culturomics, applying 17 different culture methods.

Results and conclusions. Several different bacteria were isolated from donors. In two donors 4 potentially pathogenic bacteria were isolated; this suggests the use of culturomics for donors' screening or for selection of bacteria to infuse.

\section{Introduction}

Human gut microbiota consists of approximately $10^{13}-10^{14}$ bacteria (9). Its alteration seems to be involved in several diseases such as obesity, malnutrition, metabolic diseases and chronic inflammatory diseases. For this reason gut microbiota is considered like an organ, which in healthy humans confers metabolic role, deconjugation of bile acid, immunological effects and pathogens protection (15).

Clostridium difficile infection (CDI) is due to an alteration of microbiota homeostasis, following a long period of antibiotic therapy (6). Several intestinal complications could occur, such as recurrent CDI. Recurrent CDI is a recurrence of symptoms within days to several weeks after the first episode that occurs after discontinuation of antibiotic therapy. The risk of multiple episodes is high in patients who have already had one recurrence (5).

Faecal microbiota transplantation (FMT) is an infusion of faecal material obtained from healthy donors, we demonstrated its effectiveness in treating recurrent CDIs in a recently published clinical trial (3). Usually, after a screening to exclude microbial pathogens, feces of a healthy donor are used for FMT, without characterizing the composition of their microbiota.

In this study, we used a culturomic approach to isolate and identify the bacterial population in healthy donors' feces to be used in FMT in recurrent CDI patients.

Culturomics is a set of culture conditions originally applied to isolate bacterial populations considered unculturable. Culture conditions include: several enrichment broth media, blood culture bottles, culture on selective and non- 
selective agar media, different temperatures and times of observation from few days to months (8).

\section{Materials and methods}

All aspects of this study were approved by the Ethical Committee of "Fondazione Policlinico Universitario A. Gemelli". Written consent was obtained for all participants.

Three faecal samples obtained from three healthy donors for FMT were analysed by culturomics.

\section{Donors screening}

The donors' health conditions were assessed as previously described (3). As regards faecal samples, they were tested for $C$. difficile Toxin A/B [Liaison, DiaSorin SpA, Saluggia (VC), Italy], enteric bacteria, protozoa and helminths, VRE (vancomycin-resistant Enterococci), MRSA (methicillin-resistant Staphylococcus aureus), and Gram-negative MDR (multi-drug-resistant) bacteria, according to international guideline procedures.

Donor 1 was a 45 years old daughter, donor 2 was a 42 years old daughter and donor 3 was a 43 years old brotherin law of each recipient.

\section{Culturomics protocol}

Thirty grams of each faecal sample were dissolved and re-suspended in $30 \mathrm{~mL}$ of saline solution. After the homogenization, there was a filtration step using patented filters, included in the Parasep ${ }^{\circledR}$ Faecal Parasite Concentrators kit (Apacor Limited, Sapphire Center, Wokingham, England). In these filters, large particles are rejected without occluding the $425 \mu \mathrm{m}$ pores, according to manufacturer's instructions.

The filtered $30 \mathrm{~mL}$ were divided into three aliquots of nearly $10 \mathrm{~mL}$ each and then centrifuged at $3500 \mathrm{~g}$ for $10 \mathrm{~min}-$ utes. Supernatants were discarded and each one of the three pellets was resuspended: one in $5 \mathrm{~mL}$ of Brain Hearth Infusion (BHI, Oxoid Deutschland GmbH, Wesel, Germany), one in $5 \mathrm{~mL}$ of supplemented Brucella Broth (BB, Remel INC., Lenexa, USA) and the last in $5 \mathrm{~mL}$ of rumen fluid.
Rumen fluid was obtained from a cow fed with alfalfa-hay, before slaughtering. We modified the protocol described by Santschi et al. (16); briefly rumen fluid was filtered through two layers of gauzes and it was stored refrigerated overnight at $4^{\circ} \mathrm{C}$ to settle down particulate material. Supernatant was submitted to filter sterilization through $0.22 \mu \mathrm{m}$ Minisart ${ }^{\circledR}$ filters (Sartorius AG, Weender Landstr. Goettingen, Germany)

BHI suspension was divided into two aliquots of $2.5 \mathrm{~mL}$ each, which were inoculated into two bottles of paediatric blood cultures (Becton, Dickinson and Company, Benex Limited, Shannon, Ireland), four aliquots of $2.5 \mathrm{~mL}$ each were obtained from the other two suspensions inoculated into four bottles of anaerobic blood culture (Becton, Dickinson and Company, Ireland), which were incubated at $37^{\circ} \mathrm{C}$, vortexed and then $100 \mu \mathrm{L}$ plated on agar media at day 7 and day 14. Culture conditions are shown in Table 1.

\section{Identification of isolated bacteria}

Bacterial identification was performed by MALDI-TOF, Mass Spectrometry, on a Bruker MicroFlex LT (Bruker Daltonics, Billerica, MA, USA).

For plate extractions, 1 to 4 colonies from subcultures were spotted on the target plate. Each spot was overlaid with $1 \mu \mathrm{L}$ of alpha-cyano-4-hydroxycinnamic acid ( $\alpha$-CHCA).

Spectra were acquired from 1000 laser shots at a minimum intensity threshold of 30 arbitrary units (A.U.). Plate calibration was performed on each run before spectral acquisition using a mixture of two proteins contained in Bacterial Test Standard (BTS) (Bruker Daltonics).

The BTS has been developed for the Quality Control (QC) process of the MALDI Biotyper system. Its specific composition covers the entire mass range of proteins used by MALDI Biotyper for precise identification of microorganisms, making BTS ideal for use with this mass spectrometry system. When BTS is used, MALDI Biotyper performs an automatic QC before each identification run. The QC process includes calibration of the mass spectrometer, a laser setting check and evaluation of spectrum quality. System performance is finally confirmed by achieving Escherichia coli identification, which must pass minimum

\section{Table 1. Media and culture conditions.}

1. Pre-incubation in paediatric blood culture bottle with rumen fluid and then $5 \%$ sheep blood, Chocolate and McConkey agar under aerobic, aerobe with $2.5 \% \mathrm{CO}_{2}$ and microaerophilic conditions at $37^{\circ} \mathrm{C}$

2. Pre-incubation in anaerobic blood culture bottle with rumen fluid and then Columbia and Schaedler agar under anaerobic conditions at $37^{\circ} \mathrm{C}$

3. Pre-incubation in paediatric blood culture bottle with Brucella broth and then $5 \%$ sheep blood, Chocolate and McConkey agar under aerobic, aerobe with $2.5 \% \mathrm{CO}_{2}$ and microaerophilic conditions at $37^{\circ} \mathrm{C}$ agar under aerobic conditions at $37^{\circ} \mathrm{C}$

4. Pre-incubation in anaerobic blood culture bottle with Brucella broth and then Columbia and Schaedler agar under anaerobic conditions at $37^{\circ} \mathrm{C}$

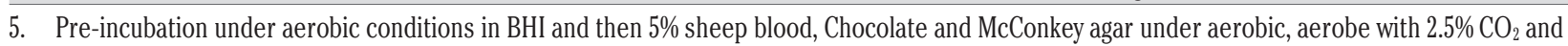
microaerophilic conditions at $37^{\circ} \mathrm{C}$ agar under aerobic conditions at $37^{\circ} \mathrm{C}$

6. Pre-incubation in anaerobic blood culture bottle with stool filtered at $5 \mathrm{~m}$ and then Columbia and Schaedler agar under anaerobic conditions at $37^{\circ} \mathrm{C}$

7. Pre-incubation in paediatric culture bottle with stool filtered at $5 \mathrm{~m}$ and then $5 \%$ sheep blood under aerobic conditions at $37^{\circ} \mathrm{C}$

8. Pre-incubation in paediatric blood culture bottle with $5 \mathrm{~mL} \mathrm{BHI}$ and then $5 \%$ sheep blood, Chocolate and McConkey agar under aerobic, aerobe with $2.5 \%$ $\mathrm{CO}_{2}$ and microaerophilic conditions at $37^{\circ} \mathrm{C}$ agar under aerobic conditions at $37^{\circ} \mathrm{C}$

9. Pre-incubation in anaerobic blood culture bottle with $5 \mathrm{~mL} \mathrm{BHI}$ and then Columbia and Schaedler agar under anaerobic conditions at $37^{\circ} \mathrm{C}$ 
performance criteria. The result is documented on each identification run report.

Plate calibration met acceptable specifications when at least seven of the eight proteins were detected within 150 ppm of the in last fit parameter, analysed with Flex Analysis software (Version 3.4; Bruker Daltonics).

\section{Results}

Following bacterial species were isolared from donor 1 Actinomyces neuii, Actinomyces turicensis, Alistipes shahii, Bacteroides uniformis, Bacteroides intestinalis, Bacteroides salyersiae, Bifidobacterium adolescentis, Blautia coccoides, Clostridium aldenense, Clostridium scindens, Enterococcus faecium, Escherichia coli, Eggerthella lenta, Nocardia arthritidis, Parabacteroides distasonis, Parabacteroides johnsonii and Streptococcus anginosus.

In donor 2 Bacteroides intestinalis, Bacteroides thetaiotaomicron, Bacteroides uniformis, Citrobacter amalonaticus, Clostridium bifermentans, Clostridium butyricum, Clostridium sordellii, Clostridium tertium, Enterococcus faecalis, Enterococcus faecium, Escherichia coli, Flavonifractor plautii, Lactobacillus rhamnosus, Parabacteroides distasonis and Pseudomonas agarici were detected

Finally, in donor 3 we found Bacteroides uniformis, Bacteroides vulgatus, Clostridium bifermentans, Clostridium clostridioforme, Clostridium perfringens, Clostridium tertium, Enterococcus faecium, Escherichia coli, Klebsiella oxytoca, Parabacteroides distasonis and Proteus mirabilis (Table 2).

\section{Discussion}

C. difficile is a Gram-positive, spore-forming, anaerobic pathogen, which is acquired via faecal-oral route, through ingestion of the bacterial spores. After reaching the anaerobic gut niche of susceptible patients, the spores germinate into metabolically active vegetative cells that produce two major exotoxins, Toxin A (TcdA) and Toxin B (TcdB) (19). Some strains of $C$. difficile produce a third toxin, CDT $(C$. difficile transferase), which may also be involved in the pathogenic process (1). These strains have been associated with severe forms of disease in humans, even if the roles of these toxins in pathogenesis aren't clear yet (2).

Recurrent CDI is associated with prolonged antibiotic therapy that could destroy the normal intestinal flora, enhancing a decrease in colonization resistance (18). In fact the normal microbiota of adults is dominated by species of the phyla Bacteroidetes and Firmicutes (9), whereas the predominant species in patients with recurrent CDI were Proteobacteria and Verrucomicrobia, reducing dominance of Bacteroidetes (4).

The main knowledge about the composition of gut microbiota was acquired using molecular methods such as DGGE (11) and TGGE (12) analysis and recently metagenomics. For many years, several bacteria were considered non-culturable and so cultures were not used to study this microbiota, but in the last years, the cultural approach is having a rebirth with culturomics (8).

Culturomics is a laborious and time-consuming approach, which is considered very useful to study living bacteria.

Using culture methods, 90 new strains were isolated and several data were obtained about faecal bacteria in people with and without diarrhea. Langier et al. developed 18 culture methods (8) whereas Samb-Ba et al. 9 (17).

In this study we applied 17 cultural conditions at 7 and 14 days on faecal samples obtained from healthy donors for FMT. We found qualitative and quantitative diversity among the bacterial species isolated in donors; according to literature this is due to different socio-economic conditions, diet, geographic areas (5) and culture methods used (8). In fact, in absence of a standardized method, various culture conditions have been used: 212 or 18 (8) and 9 (17), with different numbers of bacterial species isolated for each sample. We were able to cultivate several strains of Enterococci different strains of Bacteroides and Clostridia. In donor 1 particular bacteria were detected: Actinomyces neuii implicated in human infections such as abscesses, ulcer infections, cellulitis, urinary tract infections, prostatitis, chorioamnionitis or invasive infections such as bacteraemia, prostheses infections, chronic osteomyelitis, peritoneal dialysis-associated peritonitis, chronic pericarditis, lymphadenitis and endophthalmitis (20); Actinomyces turicensis reported as a pathogen in endocarditis (7); Eggerthella lenta isolated in blood samples (10). C. perfringens was isolated in donor 3 and its pathogenic effects are well known: gas gangrene and food poisoning (14).

Table 2. Bacteria isolated from faecal samples of the three donors.

\begin{tabular}{cl}
\hline Donor 1 & $\begin{array}{l}\text { Actinomyces neuil; Actinomyces turicensis; Alistipes shahii; Bacteroides uniformis; Bacteroides intestinalis; Bacteroides salyersiae; Bifidobacterium } \\
\text { adolescentis; Blautia coccoides; Clostridium aldenense; Clostridium scindens; Enterococcus faecium; Escherichia coli; Eggerthella lenta; }\end{array}$ \\
& Flavonifractor plautii; Nocardia arthritidis; Parabacteroides distasonis; Parabacteroides johnsonii; Streptococcus anginosus \\
Donor 2 & $\begin{array}{l}\text { Bacteroides intestinalis; Bacteroides thetaiotaomicron; Bacteroides uniformis; Citrobacter amalonaticus; Clostridium bifermentans; Clostridium } \\
\text { butyricum; Clostridium sordellii; Clostridium tertium; Enterococcus faecalis; Enterococcus faecium; Escherichia coli; Flavonifractor plautii; } \\
\text { Lactobacillus rhamnosus; Parabacteroides distasonis; Pseudomonas agarici }\end{array}$ \\
\hline Donor 3 & $\begin{array}{l}\text { Bacteroides uniformis; Bacteroides vulgatus; Clostridium bifermentans; Clostridium clostridioforme; Clostridium perfringens; Clostridium tertium; } \\
\text { Enterococcus faecium; Escherichia coli; Klebsiella oxytoca; Parabacteroides distasonis; Proteus mirabilis }\end{array}$ \\
\hline
\end{tabular} 
Since 2013, in our Institution FMT has been used to treat recurrent CDI. FMT includes: donor screening by clinical history, blood and faecal tests to be carried out 7 days before FMT, to reveal possible infections; laboratory activities to prepare feces in solution for the infusion in patients; endoscopic procedure (3).

On FMT day, the donor carries faces for infusion, which is to be made within 6 hours from the emission. Culturomics could be applied as an additional screening step to avoid to infuse even potential pathogens, such as those above described in donor 1 and 3.

In 2013, a pilot study showed that a synthetic stool solution with cultured bacteria could be an alternative to the use of donor faecal sample in the treatment of recurrent CDI (13).

\section{Conclusions}

In conclusion, culturomics could be a useful technique to set up an optimal synthetic stool sample which could have several advantages in terms of safety: i) known composition; ii) absence of potential infections acquired by donor during the period between screening and donation; iii) to have readyto-use bacterial solutions for emergency infusions.

\section{References}

1. Aktories K, Wegner A. Mechanisms of the cytopathic action of actin-ADP-ribosylating toxins. Mol Microbiol 1992;6:2905-8.

2. Awad MM, Johanesen PA, Carter GP, et al. Clostridium difficile virulence factors: insights into an anaerobic spore-forming pathogen. Gut Microbes 2014;5:579-93.

3. Cammarota G, Masucci L, Ianiro G, et al. Randomised clinical trial: faecal microbiota transplantation by colonoscopy vs. vancomycin for the treatment of recurrent Clostridium difficile infection. Aliment Pharmacol Ther 2015;41:835-43.

4. Chang JY, Antonopoulos DA, Kalra A, et al. Decreased diversity of the faecal microbiome in recurrent Clostridium difficile associated diarrheal. J Infect Dis 2008;197:435-8.

5. Daotong L, Pan W, Pengpu W, et al. The gut microbiota: a treasure for human health. Biotechnol Adv 2016;34: 1210-24.

6. Denève C, Janoir C, Poilane I, et al. New trends in
Clostridium difficile virulence and pathogenesis. Int $\mathbf{J}$ Antimicrob Ag 2009;33:S24-8.

7. Kottam A, Kaur R, Bhandare D, et al. Actinomycotic endocarditis of the eustachian valve: a rare case and a review of the literature. Tex Heart Inst J 2015;42:44-9.

8. Lagier JC, Hugon P, Khelaifia S, et al. The rebirth of culture in microbiology through the example of colturomics to study human gut microbiota. Clin Microbiol Rev 2015;28:237-64.

9. Ley RE, Peterson DA, Gordon JI. Ecological and evolutionary forces shaping microbial diversity in the human intestine. Cell 2006;124:837-48.

10. Liderot K, Ratcliffe $P$, Lüthje $P$, et al. Microbiological diagnosis of Eggerthella lenta blood culture isolates in a Swedish tertiary hospital: rapid identification and antimicrobial susceptibility profile. Anaerobe 2016;38:21-4.

11. Mai V, Morris JG Jr. Colonic bacterial flora: changing understandings in the molecular age. J Nutr 2004;134: 459-64.

12. McCartney AL. Application of molecular biological methods for studying probiotics and the gut flora. $\mathrm{Br} \mathrm{J}$ Nutr 2002;88:S29-37.

13. Petrof EO, Gloor GB, Vanner SJ, et al. Stool substitute transplant therapy for the eradication of Clostridium difficile infection: RePOOPulating the gut. Microbiome 2013;1:3.

14. Ohtani K, Shimizu T. Regulation of toxin production in Clostridium perfringens. Toxins (Basel) 2016;8.

15. Quigley EMM. Gut bacteria in health and disease. Gastroenterol Hepatol 2013;9:560-9.

16. Santschi DE, Chiquette J, Berthiaume R, et al. Effects of methods of collection and sample preparation on the concentrations of B-vitamins in ruminal fluid of dairy cows Can J Anim Sci 2005;85:417-20.

17. Samb-Ba B, Mazenot C, Gassama-Sow A, et al. MALDI-TOF Identification of the human gut microbiome in people with and without diarrheal in Senegal. Plos One 2014;9:e87419.

18. Stuart J. Recurrent Clostridium difficile infection: causality and therapeutic approaches. Int J Antimicrob Ag 2009;33:S33-6.

19. Vedantam G, Clark A, Chu M, et al. Clostridium difficile infection: toxins and non-toxin virulence factors, and their contributions to disease establishment and host response. Gut Microbes 2012;3:121-34.

20. Zelyas N, Gee S, Nilsson B, et al. Infections caused by Actinomyces neuii: a case series and review of an unusual bacterium. Can J Infect Dis Med Microbiol 2016;2016:1-7. 